\title{
Abkürzungen und Zitierweisen
}

\section{Abgekürzt zitierte Werke}

ACO Schwartz, Eduard, Hg. Acta Conciliorum Oecumenicorum. Tomus primus. Berlin/Leipzig: Walter de Gruyter, 1927-1930.

CPG Geerard, Mauritius, Hg. Clavis Patrum Graecorum. Bde. 1-5 und Supplement. Turnhout: Brepols, 1974-2003.

CSCO Chabot, Jean-Baptiste u.a., Hg. Corpus scriptorum Christianorum orientalium. Louvain: Peeters, $1903 \mathrm{ff}$.

Lampe Lampe, Geoffrey W. H., Hg. A Patristic Greek Lexicon. Oxford: Clarendon Press, 1961-1968.

LSJ Liddell, Henry G. und Robert Scott u.a. Greek-English Lexicon. Reprint with a revised supplement. Oxford: Clarendon Press, ${ }^{9} 1996$.

PG Migne, Jacques P., Hg. Patrologiae cursus completus, Series Graeca. Paris: Paul Geuthner, 1857-1866.

PL Migne, Jacques P., Hg. Patrologiae cursus completus, Series Latina. Paris: Garnier frères, 1841-1905.

PLRE Jones, Arnold H. M., John R. Martindale und John Morris, Hg. The Prosopography of the Later Roman Empire. 3 Bde. Cambridge: Cambridge University Press, 1971-1992.

SC Lubac, Henri de u.a., Hg. Sources chrétiennes. Paris: Les Éditions du Cerf, 1941ff.

TLG Brunner, Theodore F. u.a., Director. Thesaurus Linguae Graecae, A Digital Library of Greek Literature. Irvine: University of California, $1976 \mathrm{ff}$.

\section{Häufig zitierte Ausgaben und ihre Zitierweise}

\section{Julian}

Bidez 1924 (ND 2003):

Bidez, Joseph, Hg. L'Empereur Julien, EFuvres complètes. Tome I, $2^{\mathrm{e}}$ partie: Lettres et fragments. Collection des Universités de France 22. Paris: Les Belles Lettres.

Ep. Nr. Bidez, S.,Z.-Z.

\section{Masaracchia 1990:}

Masaracchia, Emanuela, Hg. Giuliano Imperatore, Contra Galilaeos. Testi e commenti 9. Roma: Edizioni dell'Ateneo.

CG fr. Nr.,Z.-Z. Mas.

Nesselrath 2015:

Nesselrath, Heinz-Günther, Hg. Iulianus Augustus, Opera. Bibliotheca scriptorum Graecorum et Romanorum Teubneriana 2018. Berlin/Boston: Walter de Gruyter.

Nesselrath S.,Z.-Z.

Neumann 1880:

Neumann, Johannes, Hg. Iuliani imperatoris librorum contra Christianos quae supersunt. Collegit, recensuit, prolegomenis instruxit C.I. N. Insunt Cyrilli Alexandrini fragmenta syriaca ab Eber- 
hardo Nestle edita. Scriptorum Graecorum qui Christianam impugnaverunt religionem quae supersunt fasc. 3. Leipzig: Teubner.

CG Neumann S.,Z.-Z.

Cl Buch [arab.], fr. Neumann

\section{Kyrill}

Wenn nicht anders angegeben, werden Stellen aus Contra Iulianum (CI) nach Riedweg 2016, Kinzig/Brüggemann 2017 und Kaufhold 2017 zitiert.

Boulnois u.a. 2016:

Boulnois, Marie-Odile u.a., Hg. Cyrille d'Alexandrie, Contre Julien. Tome II (livres III-V). Introduction et annotation Marie-Odile Boulnois. Texte grec Christoph Riedweg (GCS NF 20). Traduction Jean Bouffartigue (†), Marie-Odile Boulnois et Pierre Castan. Sources Chrétiennes 582. Paris: Les Éditions du Cerf.

C) Buch[röm.],§,Z.-Z. (SC Bd.-Nr., S.)

Burguière/Évieux 1985:

Burguière, Paul und Pierre Évieux, Hg. Cyrille d'Alexandrie, Contre Julien. Tome I: Livres I et II. Introduction, texte critique, traduction et notes par Paul Burguière et Pierre Évieux. Sources Chrétiennes 322. Paris: Les Éditions du Cerf.

C) Buch[röm.],§,Z.-Z. (SC Bd.-Nr., S.)

Évieux u.a. 1991:

Évieux, Pierre u.a., Hg. Cyrille d'Alexandrie, Lettres Festales I-VI. Vol. 1. Introduction générale par Pierre Évieux, introduction critique, texte grec par William H. Burns, traduction et annotation par Louis Arragon, Marie-Odile Boulnois, Pierre Évieux, Marguerite Forrat, Bernard Meunier. Sources Chrétiennes 372. Paris: Les Éditions du Cerf.

LF Nr.,§,Z.-Z. (SC Bd.-Nr., S.)

Burns u.a. 1993:

Burns, William H. u.a., Hg. Cyrille d'Alexandrie, Lettres Festales VII-XI. Vol. 2. Texte grec par William H. Burns, traduction et annotation par Louis Arragon, Pierre Évieux, Robert Monier. Sources Chrétiennes 392. Paris: Les Éditions du Cerf.

LF Nr.,§,Z.-Z. (SC Bd.-Nr., S.)

Burns u.a. 1998:

Burns, William H. u.a., Hg. Cyrille d'Alexandrie, Lettres Festales XII-XVII. Vol. 3. Texte grec par William H. Burns, traduction et annotation par Marie-Odile Boulnois, Bernard Meunier. Sources Chrétiennes 434. Paris: Les Éditions du Cerf.

LF Nr.,§,Z.-Z. (SC Bd.-Nr., S.)

Kaufhold 2017:

Kaufhold, Hubert, Hg. “Die syrischen Fragmente.” In Kyrill von Alexandrien, Werke: Band I »Gegen Julian «, Teil 2: Buch 6-10 und Fragmente. Herausgegeben von Wolfram Kinzig und Thomas Brüggemann. Syrische Fragmente herausgegeben von Hubert Kaufhold. Die Griechischen Christlichen Schriftsteller der ersten Jahrhunderte NF 21, 821-895. Berlin/Boston: Walter de Gruyter.

Cl fr. Syr. Nr., Z.-Z. 


\section{Kinzig 2016:}

Kinzig, Wolfram. “Allgemeine Einleitung: 2. Editionen, 3. Übersetzungen, 6.1.-5. Kyrill - Contra Iulianum.” In Kyrill von Alexandrien, Werke: Band I »Gegen Julian«, Teil 1: Buch 1-5. Herausgegeben von Christoph Riedweg. Mit einer allgemeinen Einleitung von Christoph Riedweg und Wolfram Kinzig. Die Griechischen Christlichen Schriftsteller der ersten Jahrhunderte NF 20, LXIV-LXXXI und CIX-CLXXIV. Berlin/Boston: Walter de Gruyter.

Kinzig/Brüggemann 2017:

Kinzig, Wolfram und Thomas Brüggemann, Hg. Kyrill von Alexandrien, Werke: Band I »Gegen Julian«, Teil 2: Buch 6-10 und Fragmente. Herausgegeben von Wolfram Kinzig und Thomas Brüggemann. Syrische Fragmente herausgegeben von Hubert Kaufhold. Die Griechischen Christlichen Schriftsteller der ersten Jahrhunderte NF 21. Berlin/Boston: Walter de Gruyter.

Cl Buch,§,Z.-Z. / Cl fr. Nr., Z.-Z.

Pusey:

Pusey, Philip E., Hg. Sancti patris nostri Cyrilli archiepiscopi Alexandrini [opera] post Pontanum et Aubertum edidit P.E. P., Bde. I-II: In XII prophetas, Bde. III-V: In D. Joannis Evangelium. Accedunt fragmenta varia necnon Tractatus ad Tiberium diaconum duo. Oxford: Clarendon 1868-1872.

Pusey Bd.[röm.],S.,Z.-Z.

Riedweg 2016:

Riedweg, Christoph, Hg. Kyrill von Alexandrien, Werke: Band I »Gegen Julian«, Teil 1: Buch 1-5. Herausgegeben von Christoph Riedweg. Mit einer allgemeinen Einleitung von Christoph Riedweg [1. Überlieferung von Kyrills Contra Iulianum, 4. Grundzüge der vorliegenden Ausgabe, 5. Julian - Contra Galilaeos, 6.6. Kyrill - Contra Iulianum: Sprache und Stil] und Wolfram Kinzig. Die Griechischen Christlichen Schriftsteller der ersten Jahrhunderte NF 20. Berlin/Boston: Walter de Gruyter.

Cl Buch,§,Z.-Z. 
